\title{
FINITELY PRESENTED LATTICES
}

\author{
RALPH FREESE AND J. B. NATION ${ }^{1}$
}

\begin{abstract}
ABSTRACr. It is shown that the generalized word problem for lattices is solvable. Moreover, one can recursively decide if two finitely presented lattices are isomorphic. It is also shown that the automorphism group of a finitely presented lattice is finite.
\end{abstract}

The solution to the word problem for a finitely presented lattice was given by J. C. C. McKinsey [9] (see also T. Evans [2]), but this still left many questions about the structure of finitely presented lattices unanswered. Our investigation was prompted by Evans's discussion of some of these problems in [3].

Throughout this paper we will fix $L$ to be a finitely presented lattice, given by the presentation

$$
L=\left\langle x_{1}, \ldots, x_{n} \mid r_{1}=s_{1}, \ldots, r_{m}=s_{m}\right\rangle
$$

where $r_{i}$ and $s_{i}$ are lattice terms in $x_{1}, \ldots, x_{n}$.

We will use the following definition of the complexity of a lattice term $w$. If $w$ is a variable, let $c(w)=0$. If $w=w_{1} \vee \cdots \vee w_{k}$, where each $w_{i}$ cannot be written as a join, or dually, let

$$
c(w)=\max \left\{c\left(w_{i}\right): i=1, \ldots, k\right\}+1 .
$$

The following lemma borrows some ideas from McKenzie [8]. Similar constructions were also used by Dean [1] and McKinsey [9].

Lemma 1. Let $L$ be a finitely presented lattice and let $d \in L$. Then there is a homomorphism $f$ of $L$ onto a finite lattice B such that $f^{-1}(f(d))=\{d\}$.

Proof. Let $X=\left\{x_{1}, \ldots, x_{n}\right\}$ and for any subset $Y$ of $L$ let $S(Y)$ be the set of all joins of finite nonempty subsets of $Y . P(Y)$ is defined dually. Let $L_{1}=(S P)^{l}(X)$ and $L_{2}=(P S)^{l}(X)$ (where $l$ will be determined shortly). Note that both are finite subsets of $L$ and thus inherit the partial ordering from $L$. Moreover, $L_{1}$ is a join-subsemilattice of $L$, and $L_{2}$ is a meet-subsemilattice of $L$. If $l \geqslant 1$, then the least and greatest elements of $L$ are in $L_{i}(i=1,2)$. Thus $L_{1}$ and $L_{2}$ are lattices. Let $\bigvee_{i}$ and $\wedge_{i}$ denote the operations of $L_{i}(i=1,2)$. Of course, $\bigvee_{1}$ agrees with $\bigvee$ on $L_{1}$, and $\wedge_{2}=\wedge$ on $L_{2}$. Also note

Presented to the Society September 21, 1978 under the title The generalized word problem for finitely presented lattices; received by the editors December 4, 1978.

AMS (MOS) subject classifications (1970). Primary 06A20; Secondary 02F47.

${ }^{1}$ The research of the authors was partially supported by NSF Grant Nos. MCS 77-01933 and MCS 76-06447 A02. 


$$
\begin{aligned}
& a \wedge_{1} b \equiv \bigvee\left\{x \in L_{1}: x<a \text { and } x<b\right\}<a \wedge b \text { for } a, b \in L_{1} \\
& a \vee_{2} b \equiv \bigwedge\left\{y \in L_{2}: y>a \text { and } y>b\right\}>a \vee b \text { for } a, b \in L_{2}
\end{aligned}
$$

Let $t$ be a lattice term such that $t\left(x_{1}, \ldots, x_{n}\right)=d$. Choose $l$ large enough so that

$$
2 l>\max \left\{c(t), c\left(r_{i}\right), c\left(s_{i}\right): i=1, \ldots, m\right\} .
$$

Then clearly $L_{1}$ and $L_{2}$ satisfy the relations $r_{i}\left(x_{1}, \ldots, x_{n}\right)=s_{i}\left(x_{1}, \ldots, x_{n}\right)$ $(i=1, \ldots, m)$, and $d \in L_{i}(i=1,2)$. Hence there are homomorphisms $f_{i}$ : $L \rightarrow L_{i}(i=1,2)$ such that $f_{i}\left(x_{j}\right)=x_{j}(j=1, \ldots, n)$. The reader can verify that

Moreover, we see that

$$
\begin{array}{ll}
f_{1}(a)=a & \text { for } a \in L_{1} \\
f_{2}(b)=b & \text { for } b \in L_{2}
\end{array}
$$

$$
\begin{array}{ll}
f_{1}(a) \leqslant a & \text { for all } a \in L \\
f_{2}(a) \geqslant a & \text { for all } a \in L .
\end{array}
$$

Indeed, let $S_{1}=\left\{y \in L: f_{1}(y) \leqslant y\right\}$. If $b, c \in S_{1}$, then

$$
f_{1}(b \wedge c)=f_{1}(b) \wedge_{1} f_{1}(c)<f_{1}(b) \wedge f_{1}(c)<b \wedge c,
$$

so $b \wedge c \in S_{1}$. Similarly $b \vee c \in S_{1}$. Thus $S_{1}$ is a sublattice of $L$, and $X \subseteq S_{1}$, so $S_{1}=L$. Hence (5) holds, and likewise (6).

Let $f: L \rightarrow L_{1} \times L_{2}$ be defined by $f(a)=\left(f_{1}(a), f_{2}(a)\right)$ for $a \in L$, and let $B=f(L)$. Clearly $B$ is finite. Since $d \in L_{1} \cap L_{2}$, by (3) and (4) we have

$$
f(d)=\left(f_{1}(d), f_{2}(d)\right)=(d, d) .
$$

If $f(a)=f(d)$, i.e., $\left(f_{1}(a), f_{2}(a)\right)=(d, d)$, then by (5) and (6) we have $d=$ $f_{1}(a)<a$ and $d=f_{2}(a)>a$, so that $d=a$. Therefore $f^{-1}(f(d))=\{d\}$.

The next theorem shows that finitely presented lattices have a property stronger than the finite separability property defined in [3].

THEOREM 2. Let $L$ be a finitely presented lattice, $U$ a sublattice of $L$, and $d \in L \backslash U$. Then there is a homomorphism from $L$ to a finite lattice with $f(d) \notin f(U)$.

Proof. The homomorphism of Lemma 1 will suffice, for if $f(d) \in f(U)$ then $f(d)=f(u)$ for some $u \in U$, implying $d=u$, a contradiction.

The generalized word problem for a finitely presented algebra $A$ in a variety $V$ asks if there is an algorithm to determine, for an arbitrary element $d \in A$ and an arbitrary finite subset $\left\{u_{1}, \ldots, u_{t}\right\}$ of $A$, if $d$ is in the subalgebra of $A$ generated by $u_{1}, \ldots, u_{t}$. By Theorem 2 and a theorem of Evans [3], we have the following.

COROLlaRY 3. The generalized word problem for lattices is solvable.

In fact, $d \in\left\langle u_{1}, \ldots, u_{t}\right\rangle$ if and only if $f(d) \in\left\langle f\left(u_{1}\right), \ldots, f\left(u_{t}\right)\right\rangle$ where $f$ : $L \rightarrow B$ is the homomorphism of Lemma 1 (which depends only on $d$ and $L$, and not $\left.u_{1}, \ldots, u_{t}\right)$. 
The isomorphism problem for a variety $V$ is to find an algorithm to decide whether two finitely presented $V$-algebras are isomorphic. Our next goal is to show that the isomorphism problem for lattices is solvable. The following lemma will be our main tool.

LEMMA 4. Let $L$ be a finitely presented lattice, and let $d \in L$. Then there is a constant $k \in \mathbf{N}$ (depending only on $d$ and the presentation of $L$ ) such that if $d=w\left(d_{1}, \ldots, d_{m}\right)$ holds in $L$ for some lattice term $w$ and $d_{1}, \ldots, d_{m} \in L$, then there is a lattice term $w^{\prime}$ with complexity $c\left(w^{\prime}\right)<k$ such that $d=$ $w^{\prime}\left(d_{1}, \ldots, d_{m}\right)$.

Proof. Consider the homomorphism $f: L \rightarrow B$ of Lemma 1 . Since $B$ is finite, there is a $k \in \mathbf{N}$ such that whenever $b=w\left(b_{1}, \ldots, b_{m}\right)$ holds for a lattice term $w$ and $b, b_{1}, \ldots, b_{m} \in B$ and $m \in \mathbf{N}$, then there is a lattice term $w^{\prime}$ of complexity at most $k$ such that $b=w^{\prime}\left(b_{1}, \ldots, b_{m}\right)$. Therefore, if $d=w\left(d_{1}, \ldots, d_{m}\right)$ in $L$, then $f(d)=w\left(f\left(d_{1}\right), \ldots, f\left(d_{m}\right)\right)=$ $w^{\prime}\left(f\left(d_{1}\right), \ldots, f\left(d_{m}\right)\right)=f\left(w^{\prime}\left(d_{1}, \ldots, d_{m}\right)\right)$ for some term $w^{\prime}$ with $c\left(w^{\prime}\right)<k$. But by Lemma 1 this implies $d=w^{\prime}\left(d_{1}, \ldots, d_{m}\right)$, as desired.

Now let $M$ be another finitely presented lattice with presentation

$$
M=\left\langle y_{1}, \ldots, y_{t} \mid r_{1}^{\prime}=s_{1}^{\prime}, \ldots, r_{p}^{\prime}=s_{p}^{\prime}\right\rangle .
$$

The reader can verify that $L$ is isomorphic to $M$ if and only if there are lattice terms $w_{i}(i=1, \ldots, n)$ in $t$ variables, and terms $u_{j}(j=1, \ldots, t)$ in $n$ variables, such that

$$
\begin{aligned}
& r_{i}\left(w_{1}\left(y_{1}, \ldots, y_{t}\right), \ldots, w_{n}\left(y_{1}, \ldots, y_{t}\right)\right) \\
& \quad=s_{i}\left(w_{1}\left(y_{1}, \ldots, y_{t}\right), \ldots, w_{n}\left(y_{1}, \ldots, y_{t}\right)\right) \text { holds in } M ; \\
& \begin{aligned}
r_{j}^{\prime}\left(u_{1}\left(x_{1}, \ldots, x_{n}\right), \ldots, u_{t}\left(x_{1}, \ldots, x_{n}\right)\right) \\
\quad=s_{j}^{\prime}\left(u_{1}\left(x_{1}, \ldots, x_{n}\right), \ldots, u_{t}\left(x_{1}, \ldots, x_{n}\right)\right) \text { holds in } L ; \\
x_{i}=w_{i}\left(u_{1}\left(x_{1}, \ldots, x_{n}\right), \ldots, u_{t}\left(x_{1}, \ldots, x_{n}\right)\right) \text { holds in } L ; \\
y_{j}=u_{j}\left(w_{1}\left(y_{1}, \ldots, y_{t}\right), \ldots, w_{n}\left(y_{1}, \ldots, y_{t}\right)\right) \text { holds in } M .
\end{aligned}
\end{aligned}
$$

Conditions (7) and (8) insure that the maps $x_{i} \rightarrow w_{i}(\mathbf{y})$ and $y_{j} \rightarrow u_{j}(\mathbf{x})$ can be extended to homomorphisms $f: L \rightarrow M$ and $g: M \rightarrow L$. Conditions (9) and (10) then insure that $g f=1_{L}$ and $f g=1_{M}$, respectively. The necessity of the conditions is apparent.

Suppose in fact that (7)-(10) hold, so that we have an isomorphism $f$ : $L \simeq M$ with $f\left(x_{i}\right)=w_{i}(\mathbf{y})$ and $f^{-1}\left(y_{j}\right)=u_{j}(\mathbf{x})$. Choose $k$ large enough so that Lemma 4 applies to $L$ with $d$ being any of the $x_{i}(i=1, \ldots, n)$, and also large enough so that it applies to $M$ with $d$ being any of the $y_{j}(j=1, \ldots, t)$. Then there exist lattice terms $w_{i}^{\prime}(i=1, \ldots, n)$ of complexity at most $k$ such that equations (9) hold when $w_{i}$ is replaced by $w_{i}^{\prime}$, i.e., such that

$$
x_{i}=w_{i}^{\prime}\left(u_{1}(\mathbf{x}), \ldots, u_{t}(\mathbf{x})\right) \text { for } i=1, \ldots, n \text {. }
$$


We claim that equations (7), (8), and (10) remain valid when $w_{i}$ is replaced by $w_{i}^{\prime}$. Checking (10), we want to know whether $y_{j}=u_{j}\left(w_{1}^{\prime}(y), \ldots, w_{n}^{\prime}(y)\right)$ holds in $M$. But since $g\left(=f^{-1}\right)$ is an isomorphism of $M$ onto $L$, these elements are equal in $M$ if and only if their images under $g$ are equal in $L$. The following shows that this is the case.

$$
\begin{aligned}
g\left(u_{j}\left(w_{1}^{\prime}(\mathbf{y}), \ldots, w_{n}^{\prime}(\mathbf{y})\right)\right)=u_{j}\left(w_{1}^{\prime}(g(\mathbf{y})), \ldots, w_{n}^{\prime}(g(\mathbf{y}))\right) \\
=u_{j}\left(w_{1}^{\prime}\left(u_{1}(\mathbf{x}), \ldots, u_{t}(\mathbf{x})\right), \ldots, w_{n}^{\prime}\left(u_{1}(\mathbf{x}), \ldots, u_{t}(\mathbf{x})\right)\right) \\
=u_{j}\left(x_{1}, \ldots, x_{n}\right) \\
=g\left(y_{j}\right),
\end{aligned}
$$

so (10) still holds. Similarly, (7) holds with $w_{i}$ replaced by $w_{i}^{\prime}$, and (8) is obviously unaffected by the change.

By a similar argument we can reduce the complexity of the terms $u_{j}$ $(j=1, \ldots, t)$ to at most $k$. Thus we have shown that there are terms $w_{i}$ $(i=1, \ldots, n)$ and $u_{j}(j=1, \ldots, t)$ such that (7)-(10) hold if and only if there are terms with complexity at most $k$ for which (7)-(10) hold.

THEOREM 5. The isomorphism problem for lattices is solvable.

Proof. The algorithm for determining if $L$ and $M$ are isomorphic is this. First determine the value of $k$ from the presentations of $L$ and $M$. Next list all sequences $\left(w_{1}, \ldots, w_{n}\right)$ of elements from $F L\left(y_{1}, \ldots, y_{t}\right)$ where $c\left(w_{i}\right)<k$ for $i=1, \ldots, n$, and all sequences $\left(u_{1}, \ldots, u_{t}\right)$ of elements from $F L\left(x_{1}, \ldots, x_{n}\right)$ where $c\left(u_{j}\right) \leqslant k$ for $j=1, \ldots, t$. There are only finitely many of each. Then check to see if (7)-(10) hold for any pair of these sequences when the corresponding terms are interpreted in $L$ and $M$. Since the word problem for finitely presented lattices is solvable, this is recursive. If such $w_{i}$ and $u_{j}$ exist, then $L \cong M$; otherwise $L \nRightarrow M$.

G. Gratzer has asked whether the automorphism group of a finitely presented lattice is always finite [6, Problem 12]. We now show that this is indeed the case. (This result was announced by Gluhov [5], but C. Herrmann has shown that his proof was invalid (see [6, Exercise 44, p. 56]).) On the other hand, A. Huhn [7] has shown that a finitely presented modular lattice may have an infinite automorphism group.

Analogously to the situation above, we see that there is an automorphism $f$ of $L$ such that $f\left(x_{i}\right)=w_{i}\left(x_{i}, \ldots, x_{n}\right)$ and $f^{-1}\left(x_{i}\right)=u_{i}\left(x_{1}, \ldots, x_{n}\right)$ for $i=$ $1, \ldots, n$ if and only if:

$$
\begin{gathered}
r_{i}\left(w_{1}(\mathbf{x}), \ldots, w_{n}(\mathbf{x})\right)=s_{i}\left(w_{1}(\mathbf{x}), \ldots, w_{n}(\mathbf{x})\right) \\
r_{i}\left(u_{1}(\mathbf{x}), \ldots, w_{n}(\mathbf{x})\right)=s_{i}\left(u_{1}(\mathbf{x}), \ldots, u_{n}(\mathbf{x})\right) ; \\
x_{i}=w_{i}\left(u_{1}(\mathbf{x}), \ldots, u_{n}(\mathbf{x})\right) \\
x_{i}=u_{i}\left(w_{1}(\mathbf{x}), \ldots, w_{n}(\mathbf{x})\right) .
\end{gathered}
$$

As above, if (11)-(14) hold, then there are $w_{i}^{\prime}(i=1, \ldots, n)$ of complexity 
at most $k$ such that (11)-(14) hold with $w_{i}$ replaced by $w_{i}^{\prime}$. Thus there is an automorphism $g$ of $L$ such that $g\left(x_{i}\right)=w_{i}^{\prime}\left(x_{1}, \ldots, x_{n}\right)$ and $g^{-1}\left(x_{i}\right)=$ $u_{i}\left(x_{1}, \ldots, x_{n}\right)$. Since the $u_{i}$ are unchanged, however, we have $g^{-1}=f^{-1}$ and hence $g=f$. Therefore

$$
w_{i}^{\prime}\left(x_{1}, \ldots, x_{n}\right)=w_{i}\left(x_{1}, \ldots, x_{n}\right)
$$

holds in $L$. This shows that there are only finitely many candidates for the images of the generators under an automorphism of $L$.

TheOREM 6. If $L$ is a finitely presented lattice, then $\operatorname{Aut}(L)$ is a finite group.

Notice that this theorem combined with Huhn's result cited above gives a (rather indirect) proof of the result of Evans and Hong [4] that the free modular lattice on four generators is not a finitely presented lattice.

\section{REFERENCES}

1. R. A. Dean, Component subsets of the free lattice on $n$ generators, Proc. Amer. Math. Soc. 7 (1956), 220-226.

2. T. Evans, The word problem for abstract algebras, J. London Math. Soc. 26 (1951), 64-71.

3. W_ Word problems, Bull. Amer. Math. Soc. 84 (1978), 789-802.

4. T. Evans and D. X. Hong, The free modular lattice on four generators is not finitely presentable, Algebra Universalis 2 (1972), 284-285.

5. M. M. Gluhov, On the problem of isomorphism of lattices, Dokl. Akad. Nauk SSSR 132 (1960), 254-256; English transl., Soviet Math. Dokl. 1 (1960), 519-522.

6. G. Gratzer, Lattice theory: First concepts and distributive lattices, W. H. Freeman, San Francisco, 1971.

7. A. Huhn, On G. Gratzer's problem concerning automorphisms of a finitely presented lattice, Algebra Universalis 5 (1975), 65-71.

8. R. McKenzie, Equational bases and nonmodular lattice varieties, Trans. Amer. Math. Soc. 174 (1972), 1-43.

9. J. C. C. McKinsey, The decision problem for some classes of sentences without quantifiers, J. Symbolic Logic 8 (1943), 61-76.

Department of Mathematics, Vanderbilt University, NashVille, TenNessee 37232

Current address (both authors): Department of Mathematics, University of Hawaii at Manoa, 265 The Mall, Honolulu, Hawaii 96822 